\title{
Age-Dependent Changes in Regulatory T Lymphocyte Development and Function: A Mini-Review
}

\author{
Julie Darrigues Joost P.M. van Meerwijk Paola Romagnoli \\ CPTP, Université de Toulouse, CNRS, Inserm, UPS, Toulouse, France
}

\section{Keywords}

Regulatory T lymphocytes · Development $\cdot$ Function

\begin{abstract}
The generation and function of immuno-suppressive regulatory T lymphocytes (Treg), which can differentiate in the thymus (tTreg) or in the periphery (pTreg), are regulated in an age-dependent manner. tTreg are produced at high levels in the first weeks of age, when they expand and colonize secondary lymphoid organs and peripheral tissues to protect the organism from autoimmune diseases and to promote tissue repair. Once this population of Treg is operational in the periphery, at puberty, thymic output of Treg declines, but self-reactive tTreg generated early on in life are maintained over time and play a major role in preserving homeostasis of the immune system. Extra-thymic pTreg differentiation declines later on in life. pTreg generated throughout life mainly protect the organism from chronic inflammation and the semi-allogeneic fetus from rejection. In this review, agedependent modulation of the production and function of these two populations of Treg is described.
\end{abstract}

ㄷ) 2017 S. Karger AG, Basel

\section{KARGER}

E-Mail karger@karger.com

www.karger.com/ger

\section{Introduction}

The immune system has evolved to protect the organism from invasion by many different pathogens (e.g., viruses, bacteria, and helminthes) and from the emergence of tumors. However, it can also react in an exacerbated manner against self and non-self host components, leading to the onset of autoimmune pathologies (e.g., juvenile diabetes and systemic lupus erythematosus) and chronic inflammatory diseases (e.g., Crohn disease).

Large-scale immuno-phenotyping studies in humans have recently analyzed inter- and intra-individual variations of leukocyte subsets before, during, and after immunological challenge. Surprisingly, these studies reported a relative harmony of the immune profile of each individual over several months, revealing that after a response the immunological parameters return to their initial state [1]. The immune system therefore tends to stability, as recently proposed by the equilibrium model of immunity of Eberl [2]. According to this model, different types of immune reactions (i.e., "type" 1 against intra-cellular pathogens, and "type 2" and "type 3" against large and small extra-cellular microorganisms, respectively) in-

Paola Romagnoli or Joost P.M. van Meerwijk

CPTP-INSERM UMR1043 - CNRS UMR5282 - Université Toulouse III BP3028, Toulouse cedex 3

FR-31024 Toulouse (France)

E-Mail Paola.Romagnoli@inserm.fr or Joost.van-Meerwijk@inserm.fr 
hibit each other, leading to an equilibrium between proinflammatory and anti-inflammatory responses, essential to preserve health.

$\mathrm{CD} 4{ }^{+}$regulatory $\mathrm{T}$ lymphocytes (Treg) expressing the transcription factor Foxp3 are key cellular mediators of this immune homeostasis. Through their immuno-suppressive capacity, Treg negatively control all types of immune responses. Three decades of studies have demonstrated their key role in preventing organ-specific autoimmune diseases, inflammatory diseases, and embryo reabsorption during pregnancy, as well as their potential to control the severe and life-threatening immune responses after organ and tissue transplantation [3]. Indeed, their absence in Scurfy mice and IPEX patients, caused by an X-linked recessive mutation in the Foxp3/ FOXP3 locus, leads to a lethal lympho-proliferative syndrome associated with endocrinopathy (e.g., type 1 diabetes) and enteropathy [3]. A comparable pathology is observed when Treg are depleted in neonatal or adult mice, highlighting the importance of these cells throughout the life span of the host [3].

Despite the apparent stability of the leukocyte populations within each individual, strong inter-individual variations have been reported [1]. It has been shown that 60 $80 \%$ of the immune system's inter-individual variations are due to non-heritable factors, predominantly age. A better characterization of these variations and the potential disequilibrium of the immune system with age could help us to predict the susceptibility of each individual to infectious diseases, cancer, or autoimmunity throughout life. We here report recent findings on age-related changes in Treg development and function.

\section{Intra- and Extra-Thymic Generation of Treg}

Treg develop as an independent lineage in the thymus and can also differentiate from conventional $\mathrm{T}$ lymphocytes (Tconv) in the periphery. Both thymus-derived Treg (tTreg) and peripherally induced Treg (pTreg) express the transcription factor Foxp3 necessary for their development, stability, and function [3]. Three conserved non-coding DNA sequences (CNS) of the Foxp3 locus regulate expression in tTreg and pTreg. Whereas CNS3, described as a "pioneer element," mediates chromatin opening and initiation of Foxp3 transcription, CNS2, a "memory element," stabilizes Foxp 3 expression in Treg progeny. CNS3 and CNS2 allow Foxp3 expression in both tTreg and $p$ Treg. CNS1, instead, contains a TGF- $\beta / N F A T$ response element and is mainly required for pTreg dif-

Age-Dependent Changes in Treg

Development and Function ferentiation [3]. The Foxp3 CNS regions and promoter are tightly controlled by epigenetic and transcriptional mechanisms [reviewed in 4].

In the thymus, regulatory and conventional $\mathrm{T}$ cells develop from a common hematopoietic progenitor of bone marrow origin. T-cell differentiation takes place sequentially in two distinct thymic compartments: the cortex and the medulla. In the cortex, thymocytes rearrange their loci (Tcra and Tcrb) encoding the $\alpha$ and $\beta$ chains of the heterodimeric T-cell receptor (TCR) for antigen. Thus, each thymocyte acquires its specificity. Still in the cortex, only thymocytes bearing a functional TCR, capable of interacting with peptide-major histocompatibility complex (MHC) complexes expressed by cortical thymic epithelial cells (cTEC), are positively selected, i.e., saved from programmed cell death. Developing T cells then undergo a first phase of negative selection in the cortex [5] essential to avoid that potentially auto-reactive and therefore dangerous $\mathrm{T}$ cells colonize the periphery. They then migrate to the medulla, where they interact with dendritic cells (DC), medullary TEC (mTEC), and B cells. mTEC and thymic $B$ cells express Aire, a transcription factor mediating ectopic expression of tissue antigens, hence allowing, within the thymus, the presentation of probably the full antigen diversity of peripheral tissues. Thymocytes interacting with too high avidity with medullary antigenpresenting cells undergo apoptotic cell death, are rendered anergic, or can differentiate into Treg [6]. These cortical and medullary processes ensure the neutralization of newly developed auto-specific T cells and the generation of a repertoire of Treg specific for tissue antigens [reviewed in 7]. The thymic medulla is therefore a major site for the establishment of immune tolerance to selfantigens in the organism.

Since the repertoire of tTreg, but not Tconv, is enriched in auto-specific cells, the molecular signals involved in Treg development are of considerable interest. Analysis of mice exclusively expressing a transgenic TCR revealed that tTreg develop only in the presence of their cognate (i.e., agonist) ligand, providing the first evidence that a high-avidity interaction between TCR and selecting peptide/MHC complexes is necessary for the generation of this important T-cell population [7]. In addition to TCR engagement, multiple costimulatory pathways [7] and cytokines [8] have been implicated in the differentiation and/or survival of developing tTreg.

Thymic Treg development is therefore a complex and tightly regulated process involving strong TCR signals and engagement of costimulatory molecules and cytokine receptors. It will now be important to investigate the po-

Gerontology 2018;64:28-35

DOI: $10.1159 / 000478044$ 
tential mechanisms modulating this process during aging and upon immune stimulation through, e.g., infection, nutrition and microbiota, pregnancy, or cancer.

As mentioned above, also peripheral $\mathrm{CD} 4^{+} \mathrm{Tconv}$ can differentiate into pTreg. The requirements for the in vitro differentiation of naïve $\mathrm{CD} 4^{+} \mathrm{Foxp}^{-} \mathrm{T}$ cells into $\mathrm{CD} 4^{+} \mathrm{Foxp}^{+}$pTreg are well established. Contrary to tTreg development, a weak TCR and CD28 stimulation must be received by naïve $\mathrm{CD} 4^{+} \mathrm{Foxp} 3^{-} \mathrm{T}$ cells to induce Foxp3 expression [reviewed in 9]. TGF- $\beta$, together with retinoic acid, plays a major role in the in vitro and in vivo induction of pTreg. Downstream elements of TGF- $\beta$ receptor signaling (SMAD3), retinoic acid receptor, and retinoic X receptor directly bind to CNS1 in the Foxp3 locus and induce Foxp3 expression [reviewed in 4]. In vivo, induction of pTreg is mainly observed in mucosal tissues (e.g., the small intestine or colon) and in gut-associated lymphoid tissue or Peyer's patches, where dietary and/or microbiota-derived antigens are constantly present [reviewed in 10]. In vitro, also the metabolic environment appears to control pTreg development. Thus, depletion of certain amino acids strongly favors the induction of Foxp3 expression [11]. Also short-chain fatty acids, metabolites from commensal microbiota, induce Foxp3 expression in Tconv [10]. Of interest, pTreg preferentially differentiate from $\mathrm{CD} 4^{+} \mathrm{Foxp} 3^{-} \mathrm{T}$ cells that have recently emigrated from the thymus, Qa-2 ${ }^{\text {low }}$ cells in mice, and $\mathrm{CD} 31^{+} \mathrm{T}$ cells in humans [12]. It therefore follows that several factors are involved in the peripheral differentiation of Tconv into Treg, probably reflecting multiple pathophysiological conditions in which this process is beneficial for the immune homeostasis of the organism.

\section{Functions of tTreg and pTreg}

Treg play a central role in the control of immune responses by inhibiting different actors of the immune system, such as T cells, B cells, NK cells, and antigen-presenting cells. Treg use a very wide repertoire of mechanisms to exert their immuno-suppressive functions. They inhibit immune responses via direct cell-cell interaction through expression of inhibitory cell surface molecules (e.g., CTLA-4, LAG-3); via secretion of anti-inflammatory mediators (e.g., IL-10, IL-35, TGF- $\beta$, neutralizing soluble receptors to IL-1 and TNF- $\alpha$ ); through adsorption of the lymphocyte growth factor IL-2; through cytotoxicity; and by interference with the metabolism (e.g., deprivation of essential amino acids, hydrolysis of ATP, and production and transfer of adenosine) [3].
The functions of tTreg versus pTreg and their relative contributions to immune homeostasis remain insufficiently defined. This is in large part due to a lack of specific markers that would allow the identification and distinction of pTreg and tTreg. Neuropilin-1 (Nrp-1), a semaphorin receptor, was reported to be exclusively expressed by tTreg and not by $\mathrm{pTreg}$ [13], but its usefulness to distinguish between these two populations is still controversial [14]. In fact, Nrp-1 expression is also acquired by $p$ Treg upon strong activation and inflammatory conditions [13]. To circumvent this problem, a molecular approach deleting CNS1 in the Foxp3 locus was used, leading to the generation of mice presenting a (partial) loss of pTreg but not tTreg. These animals developed chronic inflammation in the lung and stomach mucosa (e.g., airway inflammation, gastritis), but were free of autoimmune pathology [3]. These results therefore suggest a dichotomy in tTreg and pTreg function: tTreg, presumably specific for self-antigens, would protect the organism from autoimmune disease, whereas pTreg, presumably specific for non-self-antigens found in the mucosa, would avoid chronic inflammation. However, pTreg are also known to inhibit autoimmune (type 1) diabetes in mice, showing that this separation of functions of tTreg versus pTreg, if largely confirmed, is not absolute [15].

Analysis of Foxp $3^{C N S 1}$-deficient mice also highlighted a crucial role for pTreg in the induction of feto-maternal tolerance and avoidance of preeclampsia [16]. An alternative experimental approach, consisting of rescuing Foxp3-deficient mice by adoptive transfer of tTreg and/ or pTreg, showed that both populations were needed to avoid inflammation and weight loss. While tTreg and pTreg shared immuno-suppressive capacities, their TCR repertoires were not completely overlapping. The combined tTreg and pTreg TCR repertoires therefore appear important to protect from chronic inflammation [17]. Recent data demonstrated that the developing tTreg population is heterogeneous and contains at least two functionally diverse subpopulations. One of them (CD25 low GITR $^{\text {low }} \mathrm{PD}-1^{\text {low }}$ tTreg) induced the emergence of pTreg that prevented intestinal inflammation [18]. It therefore appears that tTreg and pTreg have overlapping but nonredundant functions in vivo.

\section{Aging and Treg Production}

One of the most remarkable changes of the immune system with age is the gradual reduction in size and function of the thymus. This organ starts to develop during
30
Darrigues/van Meerwijk/Romagnoli 
embryonic life, grows in childhood, and then progressively declines over time. Thymic involution is a complex process during which not only the size but also the architecture of the thymus is modified, with disappearance of a defined cortico-medullary junction and an increase in cTEC/mTEC ratio [reviewed in 19]. Multiple factors contribute to thymic atrophy, including a decreased number of bone marrow progenitors migrating to the thymus and of sex hormones; a reduction in number of thymic epithelial cell precursors; and an increase in adipose tissue [19]. Its onset varies with the strain of mice analyzed; an early start of thymic atrophy was observed in $\mathrm{DBA} / 2, \mathrm{FVB} / \mathrm{n}$, and NOD but not in C57BL/6 mice. Genetic analysis coupled with morphological studies has found that several genes are implicated in thymic involution, demonstrating that this process is genetically controlled [19].

The consequence of thymic involution is a drastic decline in thymopoiesis and T-cell output with age. T-cell production over time has been carefully measured in transgenic mice expressing GFP under the control of the Rag2 promoter [20]. The gene encoding the RAG-2 recombinase, involved in rearrangements of the Tcra and Tcrb genes, is switched off upon positive selection in the thymus, and accumulated GFP decays with a half-life of $56 \mathrm{~h}$ [21]. In this system, GFP expression therefore works as a molecular clock, measuring the time that has passed since positive selection. This allows distinguishing between newly developed $\left(\mathrm{GFP}^{+}\right)$and mature $\mathrm{T}$ cells $\left(\mathrm{GFP}^{-}\right)$in the thymus and in the periphery.

Hale et al. [20] observed a rapid decline in the proportion of recent thymic emigrant $\mathrm{CD}^{+}$and $\mathrm{CD} 8^{+} \mathrm{T}$ cells correlating with age-related thymic involution. Importantly, the efficiency of the development of $\mathrm{T}$ cells from their immature precursors did not change over time, indicating that the thymus maintains its functionality during life. Similar results were obtained in humans: recent thymic emigrants in humans are identified by the presence of T-cell receptor excision circles (TREC). TREC are extrachromosomal DNA by-products of Tcra rearrangement, generated therefore during thymic $\mathrm{T}$-cell development. $\mathrm{T}$ cells contain only a very limited number of TREC, which are diluted and eventually lost upon proliferation in the periphery. The number of TREC in the peripheral T cell pool declines with age, but TREC are still detected in old individuals. The latter observation implies that in humans, T-cell lymphopoiesis is still operational in the elderly [22]. Thymic involution is a conserved process in vertebrates, suggesting that it has an evolutionary advantage. It has indeed been proposed that reduced thymopoiesis may decrease the risk of developing $\mathrm{T}$-cell leukemia with age [23].

Age-Dependent Changes in Treg

Development and Function
Using Rag2-GFP mice, we recently investigated if the thymic production of Treg and Tconv declines with the same kinetics with age. We found that the production (i.e., the proportion of recent thymic emigrants among the population of interest) of Treg declines substantially faster and more than that of Tconv. In the thymus, the efficiency of Tconv development from their immature precursors remained constant over time, but that of Treg declined drastically with age [24]. These results imply the existence of a mechanism that differentially regulates thymic development of Tconv and Treg. Parallel to the drastic drop in Treg development early on in age, an impressive increase in the proportion of recirculating cells among thymic Treg was seen. RNA sequencing and flow cytometry analysis of these cells showed that recirculating thymic Treg display an effector/memory phenotype [24]. These activated Treg, coming back to the thymus from secondary lymphoid organs and peripheral tissues, inhibit the differentiation of new Treg (but not Tconv), probably by competing for the IL-2 required for their development $[24,25]$. Peripheral Treg reentering the thymus therefore quantitatively control the development of their precursors, and do so without affecting the differentiation of Tconv. This process may also be at work in humans. Activated and functionally differentiated mature Treg are present in the human thymus, where they potentially inhibit the development of new Treg [24].

Extra-thymic differentiation of pTreg also decreases with age. Since recent thymus emigrants are the preferential precursors of $p$ Treg [12], their generation is connected to thymic output of Tconv. As mentioned above, the thymus maintains its functionality through life but starts atrophying during childhood, leading to a decline in Tcell generation $[20,22,24]$. Peripheral Treg induction therefore presumably decreases in parallel to thymic involution and Tconv production. However, Carpentier et al. [26] have shown that differentiation of naïve Tconv from old mice into Treg is less efficient both in vitro and in vivo. The defective production of pTreg in the elderly may be due to a T-cell-intrinsic factor [26]. It appears therefore that the peripheral differentiation of pTreg drops with age because of a combination of reduced thymic output and potentially thymocyte-intrinsic factors. Unfortunately, in vivo longitudinal studies of the generation and function of pTreg are limited because of the lack of a reliable marker that identifies this population [14].

Taken together, these data show that generation of Treg is strictly controlled during life. Thymic production of tTreg drastically drops early on, while extra-thymic differentiation of pTreg is defective only in aged individ-

Gerontology 2018;64:28-35

DOI: $10.1159 / 000478044$ 
uals. tTreg and pTreg generation is therefore differentially regulated, and this difference may be linked to their biological function. One of the main roles of tTreg is to protect the organism from autoimmune diseases. Selfreactive Treg proliferate upon antigen encounter in the periphery and persist in secondary lymphoid organs and tissues. This Treg activation process is expected to further enrich the Treg repertoire in autospecific cells. It is also potentially responsible for the relatively large fraction of Treg recirculating back to the thymus. A constantly elevated thymic output of Treg throughout life would produce new Treg of "peripherally untested" specificity. These cells would uselessly dilute the tested peripheral Treg repertoire. If autospecific, they would also proliferate and thus consume considerable energy. We propose therefore that the negative feedback of Treg differentiation exerted by recirculating Treg is an energy-saving mechanism. It may also avoid that certain specificities become dominant through preferential proliferation. Since the feedback loop causes a rapid decline in Treg differentiation, the pool of peripheral tTreg specific for self-antigens - and thus particularly adapted to protect the organism from autoimmunity - appears to be shaped early on in life. The continuous generation of pTreg through adulthood, instead, would ensure control of the magnitude of T-cell responses against ever-new (variants of) pathogens. It would thus avoid chronic inflammation. Their decreased generation in the elderly, in whom immunity is strongly hampered, may be beneficial to promote immune responses to infectious agents.

\section{Aging and Treg Accumulation in Blood and Secondary Lymphoid Organs}

Although thymic and peripheral differentiation of Treg declines with age, mature Treg accumulate in the blood and secondary lymphoid organs of old individuals [reviewed in 27]. This accumulation depends on peripheral homeostatic mechanisms that are still poorly defined, and it appears to be linked to Treg phenotype/localization and inflammatory conditions [reviewed in 28]. According to their functional profile and independently of their thymic or peripheral origin, three main populations of peripheral Treg have been characterized: central Treg (cTreg), effector Treg (eTreg), and tissue-resident Treg [28]. eTreg have been reported to expand in the elderly. In contrast to cTreg, their survival depends on signals delivered by DC and ICOS but not by IL-2 [29], whose serum levels decrease with age [30]. In inflammatory con- ditions, cTreg rapidly proliferate and acquire an effector phenotype, contributing to the expansion of the pool of eTreg [29]. Age-related inflammation may therefore, via this mechanism, favor peripheral eTreg expansion. In addition, it has been proposed that with age, Treg lose expression of Bim, a pro-apoptotic molecule critical for maintaining T-cell homeostasis, leading to an accumulation of apoptosis-resistant Bim ${ }^{\text {low }}$ Treg [31]. An increase in Treg in the elderly may cause excessive suppression of immune responses and thus contribute to immunosenescence.

\section{Aging and Treg Function in Secondary Lymphoid Organs}

Not only the thymic production but also the functionality of T lymphocytes is defective in the elderly. Several reports have described reduced activation, effector functions, and tissue homing of T cells in old individuals, leading to increased vulnerability to infectious diseases [reviewed in 9].

As mentioned above, the generation of tTreg and pTreg is developmentally regulated. The thymic output of tTreg is maximal in neonates and infants and then rapidly declines [J.D., unpubl. observations]. It has been shown that tTreg generated during the perinatal period have a more diverse TCR repertoire and increased Treg effector function in comparison with Treg generated in adult mice. These special properties of perinatal tTreg are in part due to the capacity of neonate mTEC to present a wide array of self-peptides in association with MHC class II molecules. Perinatal Treg have a long life span and protect the organism from multi-organ auto-immunity throughout life [32]. By inhibiting the development of new tTreg from the first weeks of age on [24], peripheral Treg recirculating back to the thymus may contribute to the preservation of this crucial perinatal Treg population in the periphery.

Treg functionality is not lost from adulthood to old age. Treg isolated from young adult and old mice display similar suppressive activity in vitro [33]. It has even been described that old Treg, by producing more IL-10 and by inducing a greater down-regulation of costimulatory molecules on DC, are better suppressors than Treg from young adult mice [34]. Despite their intact suppressive activities, Treg from old mice display a different phenotype than Treg isolated from young adult mice. Several reports have shown an increased proportion of $\mathrm{CD} 25^{-}$ Treg in the elderly $[30,33]$. In contrast to CD $25^{+}$Treg, 


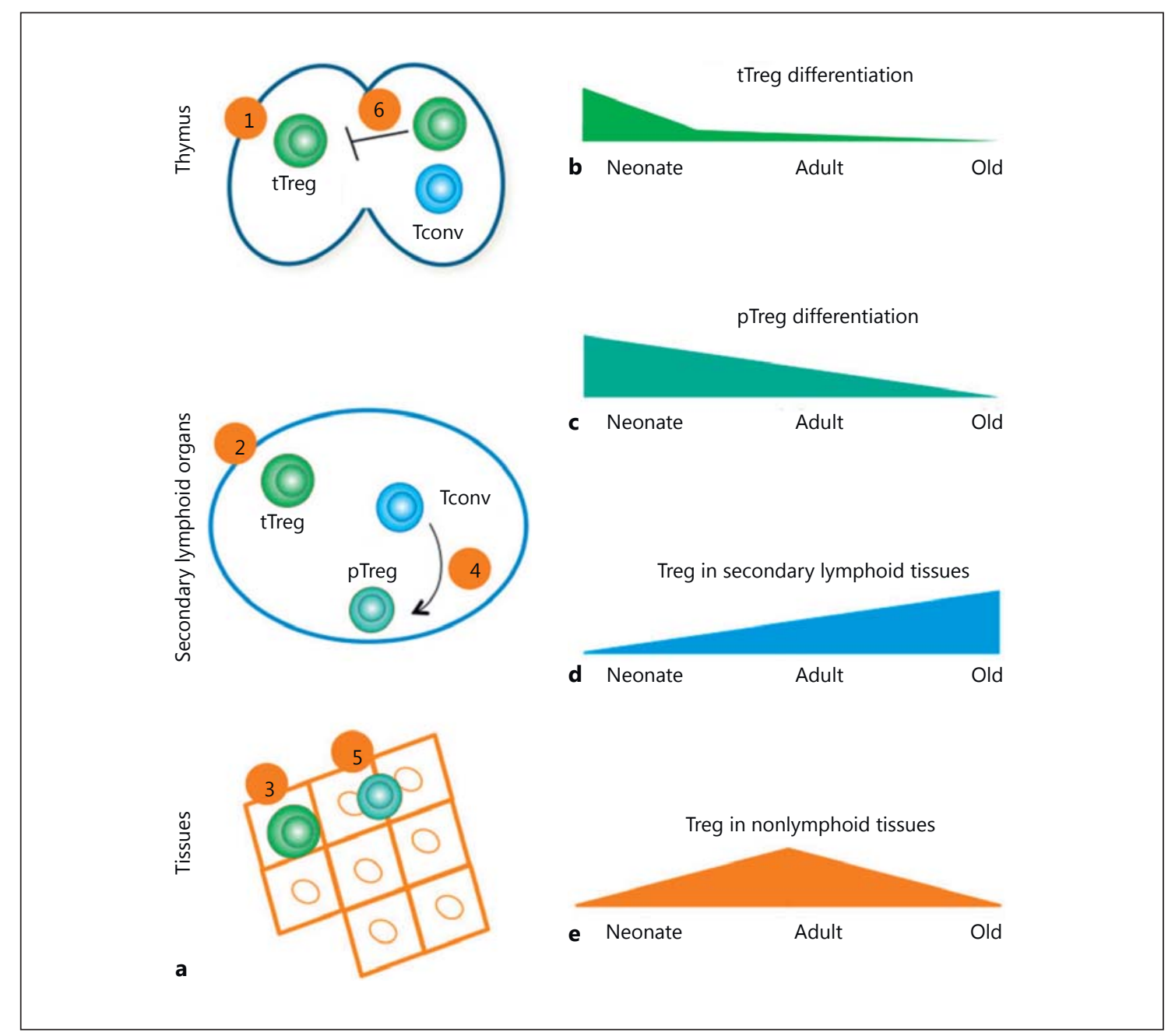

Fig. 1. Age-dependent changes in thymic development and peripheral homeostasis of regulatory T lymphocytes (Treg). a Newly developed thymus-derived Treg (tTreg) (1) migrate from the thymus to peripheral secondary lymphoid organs (2), where they perform their effector function: regulating immune system homeostasis (thereby protecting the organism from autoimmunity). Mature tTreg from secondary lymphoid organs (and potentially newly developed tTreg emigrating from the thymus) can migrate to tissues (3), acquire a tissue-specific phenotype, protect the organ from autoimmunity, and maintain tissue homeostasis by facilitating repair and regeneration (e.g., adipose tissue, muscle, and the central nervous system). Newly developed conventional $\mathrm{T}$ lymphocytes (Tconv) (4) can convert into peripherally induced Treg (pTreg) in peripheral secondary lymphoid organs and/or in tissue (5) (e.g., the colon), where they protect the host from chronic inflamma-

whose survival depends upon IL-2, survival of CD25Treg depends on IL-15. Raynor et al. [30] suggested that the age-related decrease in IL-2 levels leads to an expansion and/or accumulation of $\mathrm{CD} 25^{-}$Treg in the periphery. It remains to be determined whether decreased IL-2

Age-Dependent Changes in Treg

Development and Function tion. Activated peripheral Treg from secondary lymphoid organs and tissues migrate back to the thymus (6), where they inhibit the development of their precursors. $\mathbf{b}$ After the neonatal period, the intra-thymic differentiation of tTreg rapidly drops, due to the quickly increasing proportion of recirculating Treg in the thymus. c Also the extra-thymic differentiation of Tconv into pTreg gradually declines with age. $\mathbf{d}$ Despite the decline in tTreg and pTreg differentiation with age, Treg accumulate in secondary lymphoid organs of elderly individuals. This phenomenon may cause excessive suppression of immune responses and thus contribute to immuno-senescence. e Tissue-resident Treg accumulate from birth to adulthood, but their number drastically drops in aged individuals, leading to defective regulation of tissue/organ homeostasis and function (e.g., in muscle).

levels also affect thymic selection of Treg, favoring the emergence of IL-15-dependent tTreg with age.

In human peripheral blood, three populations of Foxp3-expressing T cells have been identified: (1) activated Treg, (2) resting Treg, and (3) cytokine-expressing 
Foxp $3^{\text {low }}$ non-regulatory T cells. Populations 1 and 2 have been shown to be highly suppressive in vitro, while population 3 produces cytokines such as IFN- $\gamma$ and IL- 2 but is not inhibitory. The ratios between these three populations change with age. In old individuals, the proportion of resting Treg drops, while the proportion of activated Treg and Foxp $3^{\text {low }}$-expressing $\mathrm{T}$ cells increases [35]. Whereas the suppressive capacities of activated/effector memory and resting/naïve Treg are comparable in young and old individuals [36], variations in the TCR repertoire expressed by Treg with age remain to be investigated.

\section{Aging and Treg Function in Tissues}

Besides protecting the organism from infectious agents, the immune system controls its homeostasis [reviewed in 37]. Tissue-resident Treg have been implicated in maintaining the integrity and functionality of tissues and organs. It has indeed been reported that Treg participate in muscle repair upon injury, that their depletion in fat induces metabolic alterations [37], and that in the central nervous system Treg display regenerative functions [38]. In the colon, Treg not only control local inflammatory responses, but they are also involved in repair processes [37]. The origin of tissue-resident Treg is still debated: muscle and fat Treg seem to derive from the thymus, while the colon Treg pool is composed of tTreg and pTreg. Tissue-resident Treg accumulate in tissues through adulthood, but their number suddenly decreases in aged individuals [37]. Although the reasons for this decline are at the moment not elucidated, the consequences on tissue repair and function are severe. In old mice, Treg do not accumulate any more in injured skeletal muscle, leading to poor muscle repair and regeneration [39]. If confirmed in humans, decreased Treg accumulation in aging individuals may therefore contribute to sarcopenia [40]. Given their relevance, future in-depth studies on the regulation and function of tissue-resident Treg will prove beneficial in the elderly.

\section{Concluding Remarks}

It appears that the generation of tTreg and pTreg, which have distinct functions in the maintenance of immune and tissue homeostasis, are differentially regulated in an age-dependent manner (Fig. 1). Thymus-derived tTreg are produced at high levels early on in life, and differentiation is rapidly inhibited by a negative feedback loop involving peripheral Treg recirculating from the periphery. Extra-thymic pTreg differentiation declines later on in life; a still unidentified T-cell-intrinsic factor appears to be involved. It will be important in the future to determine to which extent these processes are implicated in (immuno-)senescence and contribute to an increased onset of autoimmune diseases, chronic infection, cancer, sarcopenia, and potentially other pathologies in the elderly.

\section{Acknowledgements}

We thank the members of the TMIT team for critical discussions on thymic development of Treg. We apologize to many colleagues for not exhaustively quoting the primary literature, due to format restriction. This work was financially supported by the Fondation pour la Recherche Fondamentale (FRMDEQ20160334920) and the Agence Nationale pour la Recherche (ANR-16-CE15-0015-01).

\section{References}

1 Carr EJ, Dooley J, Garcia-Perez JE, et al: The cellular composition of the human immune system is shaped by age and cohabitation. Nat Immunol 2016;17:461-468.

2 Eberl G: Immunity by equilibrium. Nat Rev Immunol 2016;16:524-532.

3 Josefowicz SZ, Lu LF, Rudensky AY: Regulatory T cells: mechanisms of differentiation and function. Annu Rev Immunol 2012;30: 531-564.

4 Huehn J, Beyer M: Epigenetic and transcriptional control of Foxp3+ regulatory T cells. Semin Immunol 2015;27:10-18.
5 McCaughtry TM, Baldwin TA, Wilken MS Hogquist KA: Clonal deletion of thymocytes can occur in the cortex with no involvement of the medulla. J Exp Med 2008;205:25752584.

6 Klein L, Hinterberger M, Wirnsberger G, Kyewski B: Antigen presentation in the thymus for positive selection and central tolerance induction. Nat Rev Immunol 2009;9: 833-844.

7 Romagnoli P, van Meerwijk JP: Thymic selection and lineage commitment of $\mathrm{CD} 4^{+} \mathrm{Foxp}^{+}$ regulatory T lymphocytes. Prog Mol Biol Transl Sci 2010;92:251-277.
8 Apert C, Romagnoli P, van Meerwijk JPM: IL-2 and IL-15 dependent thymic development of Foxp3-expressing regulatory $\mathrm{T}$ lymphocytes. Protein Cell 2017, Epub ahead of print

9 Nikolich-Žugich J: Aging of the T cell compartment in mice and humans: from no naive expectations to foggy memories. J Immunol 2014;193:2622-2629.

10 Atarashi K, Umesaki Y, Honda K: Microbiotal influence on $\mathrm{T}$ cell subset development. Semin Immunol 2011;23:146-153.

Darrigues/van Meerwijk/Romagnoli 
11 Cobbold SP, Adams E, Farquhar CA, et al: Infectious tolerance via the consumption of essential amino acids and mTOR signaling. Proc Natl Acad Sci USA 2009;106:1205512060.

12 Paiva RS, Lino AC, Bergman ML, et al: Recent thymic emigrants are the preferential precursors of regulatory $\mathrm{T}$ cells differentiated in the periphery. Proc Natl Acad Sci USA 2013;110: 6494-6499.

13 Weiss JM, Bilate AM, Gobert M, et al: Neuropilin 1 is expressed on thymus-derived natural regulatory $\mathrm{T}$ cells, but not mucosa-generated induced Foxp $3^{+} \mathrm{T}$ reg cells. J Exp Med 2012;209:1723-1742, S1.

14 Szurek E, Cebula A, Wojciech L, et al: Differences in expression level of Helios and neuropilin-1 do not distinguish thymus-derived from extrathymically-induced $\mathrm{CD} 4^{+} \mathrm{Foxp}^{+}$ regulatory $T$ cells. PLoS One 2015;10: e0141161.

15 Sun J, Furio L, Mecheri R, et al: Pancreatic $\beta$-cells limit autoimmune diabetes via an immunoregulatory antimicrobial peptide expressed under the influence of the gut microbiota. Immunity 2015;43:304-317.

16 Samstein RM, Josefowicz SZ, Arvey A, et al: Extrathymic generation of regulatory $\mathrm{T}$ cells in placental mammals mitigates maternalfetal conflict. Cell 2012;150:29-38.

17 Haribhai D, Williams JB, Jia S, et al: A requisite role for induced regulatory $\mathrm{T}$ cells in tolerance based on expanding antigen receptor diversity. Immunity 2011;35:109-122.

18 Wyss L, Stadinski BD, King CG, et al: Affinity for self antigen selects $T_{\text {reg }}$ cells with distinct functional properties. Nat Immunol 2016;17: 1093-1101.
19 Dooley J, Liston A: Molecular control over thymic involution: from cytokines and microRNA to aging and adipose tissue. Eur J Immunol 2012;42:1073-1079.

20 Hale JS, Boursalian TE, Turk GL, Fink PJ: Thymic output in aged mice. Proc Natl Acad Sci USA 2006; 103:8447-8452.

21 McCaughtry TM, Wilken MS, Hogquist KA: Thymic emigration revisited. J Exp Med 2007; 204:2513-2520.

22 Jamieson BD, Douek DC, Killian S, et al: Generation of functional thymocytes in the human adult. Immunity 1999;10:569-575.

23 Boehm T, Swann JB: Thymus involution and regeneration: two sides of the same coin? Nat Rev Immunol 2013;13:831-838.

24 Thiault N, Darrigues J, Adoue V, et al: Peripheral regulatory $\mathrm{T}$ lymphocytes recirculating to the thymus suppress the development of their precursors. Nat Immunol 2015;16:628-634.

25 Weist BM, Kurd N, Boussier J, et al: Thymic regulatory $\mathrm{T}$ cell niche size is dictated by limiting IL-2 from antigen-bearing dendritic cells and feedback competition. Nat Immunol 2015;16:635-641.

26 Carpentier M, Chappert P, Kuhn C, et al: Extrathymic induction of Foxp $3^{+}$regulatory $\mathrm{T}$ cells declines with age in a T-cell intrinsic manner. Eur J Immunol 2013;43:2598-2604.

27 Jagger A, Shimojima Y, Goronzy JJ, Weyand $\mathrm{CM}$ : Regulatory $\mathrm{T}$ cells and the immune aging process: a mini-review. Gerontology 2014;60: 130-137.

28 Liston A, Gray DH: Homeostatic control of regulatory T cell diversity. Nat Rev Immunol 2014;14:154-165.

29 Smigiel KS, Richards E, Srivastava S, et al: CCR7 provides localized access to IL-2 and defines homeostatically distinct regulatory $\mathrm{T}$ cell subsets. J Exp Med 2014;211:121-136.

30 Raynor J, Sholl A, Plas DR, et al: IL-15 fosters age-driven regulatory $\mathrm{T}$ cell accrual in the face of declining IL-2 levels. Front Immunol 2013; 4:161.
31 Chougnet CA, Tripathi P, Lages CS, et al: A major role for Bim in regulatory $\mathrm{T}$ cell homeostasis. J Immunol 2011;186:156-163.

32 Yang S, Fujikado N, Kolodin D, et al: Immune tolerance. Regulatory $\mathrm{T}$ cells generated early in life play a distinct role in maintaining selftolerance. Science 2015;348:589-594.

33 Nishioka T, Shimizu J, Iida R, et al: $\mathrm{CD}^{+} \mathrm{CD} 25^{+} \mathrm{Foxp}^{+} \mathrm{T}$ cells and $\mathrm{CD} 4^{+} \mathrm{CD} 25^{-}$ Foxp $3^{+} \mathrm{T}$ cells in aged mice. J Immunol 2006; 176:6586-6593.

34 Garg SK, Delaney C, Toubai T, et al: Aging is associated with increased regulatory T-cell function. Aging Cell 2014;13:441-448.

35 Miyara M, Yoshioka Y, Kitoh A, et al: Functional delineation and differentiation dynamics of human $\mathrm{CD}^{+} \mathrm{T}$ cells expressing the FoxP3 transcription factor. Immunity 2009; 30:899-911.

36 Santner-Nanan B, Seddiki N, Zhu E, et al: Accelerated age-dependent transition of human regulatory T cells to effector memory phenotype. Int Immunol 2008;20:375-383.

37 Panduro M, Benoist C, Mathis D: Tissue Tregs. Annu Rev Immunol 2016;34:609-633.

38 Dombrowski Y, O'Hagan T, Dittmer M, et al: Regulatory $\mathrm{T}$ cells promote myelin regeneration in the central nervous system. Nat Neurosci 2017;20:674-680.

39 Kuswanto W, Burzyn D, Panduro M, et al: Poor repair of skeletal muscle in aging mice reflects a defect in local, interleukin-33-dependent accumulation of regulatory $\mathrm{T}$ cells. Immunity 2016;44:355-367.

40 Cruz-Jentoft AJ, Baeyens JP, Bauer JM, et al: Sarcopenia: European consensus on definition and diagnosis: Report of the European Working Group on Sarcopenia in Older People. Age Ageing 2010;39:412-423.
Age-Dependent Changes in Treg

Development and Function
Gerontology 2018;64:28-35

DOI: $10.1159 / 000478044$ 